\title{
Connected Rotation-invariant Size-Shape Granulometries
}

\author{
Erik R. Urbach, Jos B.T.M. Roerdink and Michael H.F. Wilkinson \\ Institute of Mathematics and Computing Science, University of Groningen \\ P.O. Box 800, 9700 AV Groningen, The Netherlands \\ \{erik, roe,michael\}@cs.rug.nl
}

\begin{abstract}
In this paper we describe a rotation-invariant multiscale morphological method for texture analysis. Compared with existing methods our method has three advantages. First, it can be implemented efficiently. Furthermore, our method can be used for the computation of size and strict shape attributes, which we use for the computation of 2-D size-shape pattern spectra. Finally, our method is rotationinvariant. Although the latter can also be approximated by morphological methods by using structuring elements at different angles, this tends to be computationally intensive.
\end{abstract}

\section{Introduction}

Pattern spectra are commonly used tools for image analysis and classification [6,11], which can be computed using a technique from mathematical morphology known as granulometries [2, 9]. Intuitively, a size granulometry can be considered as a set of sieves of different grades, each allowing details of certain size classes to pass. More formally, a size granulometry consists of an ordered set of operators each of which converts an image to a new image in which features smaller than a particular size are absent. Granulometries based on shape have also been proposed [2, 14]. Granulometries are often computed using filters with structuring elements. Connected filters can also be used for this purpose $[2,10]$. These are operators which only ever merge flat zones, or change their gray level, but never split them This means no new edges are introduced.

In the following our method is discussed which is based on Salembier's Max-tree algorithm. An existing method using structuring elements is discussed briefly in section 3.2. The performance of both methods is discussed in section 4 using the application of diatom identification.

Diatoms [13] are unicellular algae with highly ornate silica shells, which consist of two halves called valves. Examples of diatom valves are shown in Fig. 1. Both the shapes of these shells and the textures or ornamentation are distinc-

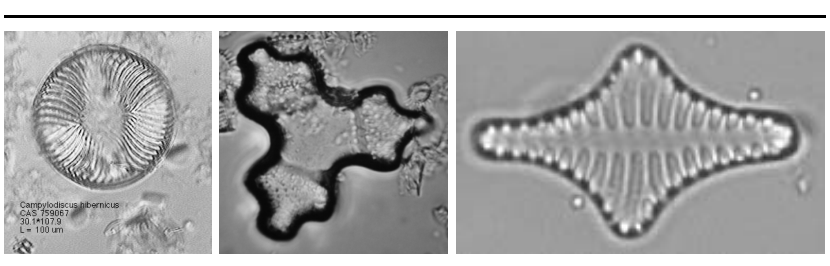

Figure 1. Some examples of diatom shells

tive characteristics of individual species or varieties. Usually, diatoms are identified manually, using both the internal texture and the outline shape. To automate the whole process of diatom identification the Automatic Diatom Identification And Classification project (ADIAC) [4] was started. The methods described in this paper compute the feature vectors from the ornamentation only.

\section{Theory}

A size granulometry is a set of operators $\left\{\alpha_{r}\right\}$ with $r$ from some totally ordered set $\Lambda$ (usually $\Lambda \subset \mathbb{R}$ or $\mathbb{Z}$ ), with the following three properties

$$
\begin{aligned}
\left(\alpha_{r}(f)\right)(x) & \leq f(x) \\
f \leq g & \Rightarrow \alpha_{r}(f) \leq \alpha_{r}(g) \\
\alpha_{r}\left(\alpha_{s}(f)\right) & =\alpha_{\max (r, s)}(f),
\end{aligned}
$$

for all $r, s \in \Lambda$. Since (1) and (2) define $\alpha_{r}$ as anti-extensive and increasing, respectively, and (3) implies idempotence, it can be seen that size granulometries are sets of openings.

Let $f_{\lambda}$ denote the scaling of an image $f$ by a scalar factor $\lambda$, then an operator $\phi$ is said to be scale-invariant if $\phi\left(f_{\lambda}\right)=(\phi(f))_{\lambda}$ for all $\lambda>0$. A scale-invariant operator is therefore sensitive to shape rather than to size.

A shape granulometry [14] is a set of operators $\left\{\beta_{r}\right\}$ with $r$ from some totally ordered set $\Lambda$, with the following three properties

$$
\begin{aligned}
\left(\beta_{r}(f)\right)(x) & \leq f(x) \\
\beta_{r}\left(f_{\lambda}\right) & =\left(\beta_{r}(f)\right)_{\lambda} \\
\beta_{r}\left(\beta_{s}(f)\right) & =\beta_{\max (r, s)}(f),
\end{aligned}
$$


for all $r, s \in \Lambda$ and $\lambda>0$. Thus, a shape granulometry consists of operators which are anti-extensive, and idempotent, but not necessarily increasing.

Size pattern spectra were introduced by Maragos [6]. Essentially they are a histogram containing the number of pixels, or the amount of image detail, over a range of size classes. If $r$ is the scale parameter of a size granulometry, the size class of $x$ is the smallest value of $r$ for which $x \notin \alpha_{r}(X)$. Shape pattern spectra can be defined in a similar way [14]. The pattern spectrum $s_{\alpha}(f)$ is usually defined as a function of scale $r$ :

$$
\left(s_{\alpha}(f)\right)(u)=\left.\frac{\mathrm{d} \int_{\mathbf{M}}\left(\alpha_{r}(f)\right)(x) d x}{\mathrm{~d} r}\right|_{r=u},
$$

and likewise for a shape granulometry.

A flat zone $L_{h}$ of an image $f$ is defined as a connected region of constant gray level $h$, no neighbours of which have gray level $h$. At each gray level there may be multiple flat zones, which are denoted by $L_{h}^{k}$, with $k$ some index variable. Similarly, a peak component $P_{h}$ of image $f$ is a connected region in which $f(x) \geq h$ for all $x \in P_{h}$, and all neighbours of $P_{h}$ have gray level smaller than $h$. Peak component $P_{h}^{k}$ can also be defined as the $k$ th connected component of the threshold set $X_{h}(f)$ of image $f$ which is defined as $X_{h}(f)=\{x \in \mathbf{M} \mid f(x) \geq h\}$. For any image $f$, the flat-zones define a partition $\left\{\alpha_{i}\right\}$ of the image domain M. A filter $\gamma$ is connected if the partition $\left\{\beta_{j}\right\}$ defined by $\gamma(f)$ is always coarser than $\left\{\alpha_{i}\right\}$, for any image $f$ [10]. By coarser is meant that for any $\alpha_{i}$ there exists a $\beta_{j}$ such that $\alpha_{i} \subseteq \beta_{j}$.

\section{The Max-tree}

The Max-tree representation was introduced by Salembier et al. [9] as a structure to separate the filtering process in three steps: construction, filtering and restitution. It is a tree where the nodes represent sets of flat zones. The Maxtree node $C_{h}^{k}$ consists of the subset of $P_{h}^{k}$ with gray level $h$. The root node represents the set of pixels belonging to the background, that is the set of pixels with the lowest intensity in the image. The Max-tree is a rooted tree: each node has a pointer to its parent, i.e. the nodes corresponding to the components with the highest intensity are the leaves (see Fig. 2). The Max-tree can be used for attribute openings or thinnings. During the construction phase, the Maxtree is built from the flat zones of the image. The nodes in a Max-tree contain one or more attributes that describe certain properties of the peak components represented by those nodes. These attributes can be used for image filtering or analysis. Besides size attributes like the area of a component, scale-invariant shape attributes can also be used. By requiring a shape attribute to be scale-invariant, we ensure it is a strict shape attribute and thus insensitive to size. Exam-

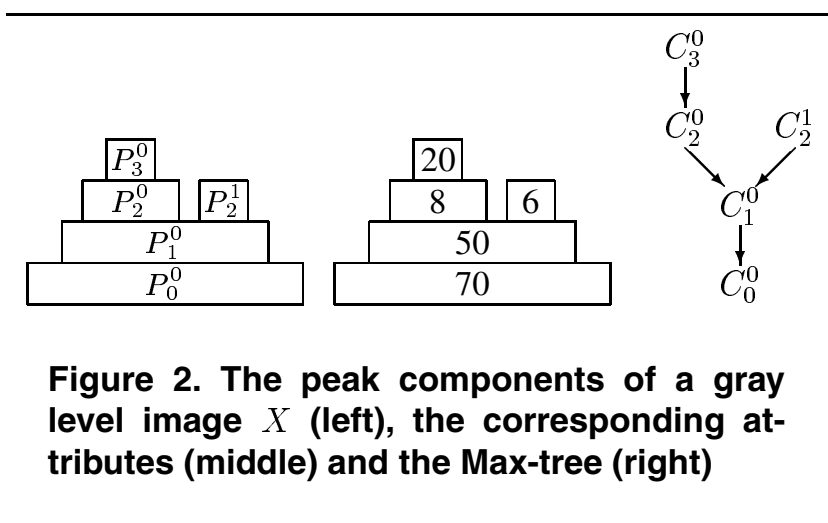

ples of scale-invariant shape attributes are many. Obvious choices are $P^{2} / A$, with $P$ the perimeter, and $A$ the area of a component, or $I / A^{2}$, which is the ratio of the moment of inertia $I$ to the square of the area. These attain minimal values for circular discs, and increase as the objects become more elongated. For computational reasons, the latter shape attribute is used, because both moment of inertia and area can easily and accurately be computed incrementally, whereas that is more difficult with perimeter. A fuller discussion is given in [2, 9].

After this, the tree is processed during the filtering phase. Based on the criterion value $T\left(P_{h}^{k}\right)$ of a node $C_{h}^{k}$, the algorithm takes a decision on whether to preserve or to remove it. By using non-increasing attributes, the property of size attributes that the attribute value of a parent is always larger than the attribute values of its children does not necessarily hold. This means a rule has to be defined how a Max-tree should be filtered with non-increasing criteria, i.e. which nodes should be removed when some do not satisfy the criterion. We used the Subtractive rule [14], which removes a node $C_{h}^{k}$ if $T\left(P_{h}^{k}\right)<r$, in which case its pixels are lowered in gray level to the highest ancestor which meets $T$ and its descendents are lowered by the same amount as $C_{h}^{k}$ itself.

Fig. 2 shows the peak components of a 1-D grayscale image, their attribute values, and the corresponding Max-tree. It has been shown that the subtractive rule works best for decomposition [14]. For this reason, we have chosen to use it in the computation of shape pattern spectra.

\subsection{2-D Shape-size pattern spectra}

Suppose we have a size granulometry $\left\{\alpha_{r}\right\}$ with $r$ from some finite index set $\Lambda_{r}$, and a shape granulometry $\left\{\beta_{s}\right\}$ with $s$ from some index set $\Lambda_{s}$. With these granulometries, we can compute a 2-D pattern spectrum, similar to that of Ghosh and Chanda [5]. We can then store a 2-D pattern spectrum, which contains both shape and scale information in a 2-D array $S$, in which $S(r, s)$ contains the sum of gray levels of those features in the image which fall in the size 
class between $r^{-}$and $r$, and shape class between $s^{-}$and $s$. Note that $r^{-}$and $s^{-}$are the members of $\Lambda_{r}$ and $\Lambda_{s}$ immediately preceding $r$ and $s$, respectively. The computation of a 2-D pattern spectrum from an image is performed as follows:

- Set all elements of the array $S$ to zero.

- Compute a Max-tree according to the algorithm in [9].

- As the Max-tree is built, compute the area $A\left(P_{h}^{k}\right)$ and moment of inertia $I\left(P_{h}^{k}\right)$ of each node.

- For each node $C_{h}^{k}$ :

- Compute the size class $r$ from the area $A\left(P_{h}^{k}\right)$.

- Compute the shape class $s$ from $\frac{I\left(P_{h}^{k}\right)}{A^{2}\left(P_{h}^{k}\right)}$.

- Compute the gray level difference $\delta_{h}$ between the current node and its parent.

- Add the product of $\delta_{h}$ and $A\left(P_{h}^{k}\right)$ to $S(r, s)$.

What remains to be specified is how to transform size and shape attributes to size and shape classes. Let $v$ be the attribute value, $c$ the class, $N_{c}$ the number of classes, and $D_{0}$ and $D_{1}$ the lower and upper bound of the range of interest of the attribute value, respectively. Because the area is a nonlinear measure of the size, when compared to width, and because most of the texture information is concentrated in the smaller peak components, we wish to have finer bins for low attribute values than high. Therefore, we compute the class $c$ from

$$
c=\frac{\left.\log _{2}(v)-\log _{2}\left(D_{0}\right)\right)}{\log _{2}\left(D_{1}\right)-\log _{2}\left(D_{0}\right)} N_{c}
$$

In our case the size classes run from $D_{0}=1$ pixel to $D_{1}=1550^{2}$ pixels (the largest image we could handle in our software). For the shape parameter $D_{0}=1 / 2 \pi$ (theoretical minimum), and $D_{1}=52$; in both cases two values were chosen for $N_{c}: 4$ and 8 . The resulting $4 \times 4,4 \times 8$, and $8 \times 4$ pattern spectra are then mapped in lexicographic order into $1-\mathrm{D}$ vectors.

Until now the assumption was made that pattern spectra were based on 2-D size-shape granulometries. However, many images contain both bright and dark image details. To obtain information about the dark patterns, anti-size and anti-shape granulometries are required, which are based on closings and thickenings respectively. Instead of designing new algorithms for the computation of pattern spectra based on these anti-size and anti-shape granulometries, these were implemented as the computation of pattern spectra of inverted images. As can be expected, the best performance was obtained by the concatenation of the down-sized vector $v_{n}$ of the normal image with the down-sized vector $v_{i}$ of the inverted image to a single feature vector $v_{r}$.

\subsection{Pattern spectra based on structuring elements}

An existing method using structuring elements was described by Dougherty et al. in [1, 12]. We implemented a method according to these papers, which computes multivariate pattern spectra using the following multivariate granulometry: $\Psi_{\mathbf{t}}(S)=\bigcup_{i=1}^{n} S \circ t_{i} B_{i}$. In order to reduce computing time, our implementation of this multivariate method uses only the horizontal and the vertical linear structuring element sequences. Two pattern spectra are computed using openings with $r=7,15,51,199$, one for the normal and one for the inverted image. Instead of using the integrated pattern spectra, we use the differentiated (in two dimensions) pattern spectra. Similar to the case of the Max-tree described earlier, the two $8 \times 4$ pattern spectra are combined to a single $1-\mathrm{D}$ feature vector containing 64 values.

\section{Results}

The performance of both the Max-tree based method and the method using structuring elements in the application of diatom identification was measured using decision trees built with the $\mathrm{C} 4.5$ algorithm [7]. To increase the stability of the decision tree classifiers, bagging [3] with 25 trees and 10 runs was used.

The original set of 781 diatom images, all of which had been aligned manually to the same orientation, consisted of 37 classes. C4.5 identified $81.0 \%, 85.1 \%$, and $85.8 \%$ of these images correctly for respectively the Max-tree $4 \times 4$, the Max-tree $4 \times 8$, and the Max-tree $8 \times 4$ method, whereas $90.8 \%$ were identified correctly using the structuring elements method.

In practice it would be undesirable for a user to have to align every image manually, which means that a method should thus be invariant to rotation. Although most of the diatom images in the set used could be aligned to have the largest axis of the valve horizontally in the image, this causes problems for diatoms with a circular or triangular contour, but with non-symmetric ornamentation patterns, like the one shown in Fig. 1(left). Another problem are diatoms shells where an orientation might be possible to compute for a given image, but where the orientation can vary w.r.t. the ornamentation between images of the same class, like the one shown Fig. 1(middle). In Fig. 3(left) the performance of both methods is shown for images rotated at different angles. As can be seen, while our method using the Max-tree algorithm with rotation-invariant attributes performs equally well for any rotation angle, the method using structuring elements only performs well for the original image set, and due to the symmetry of most diatoms, also for the image set rotated at 180 degrees. The influence of noise on the performance of both methods was also mea- 

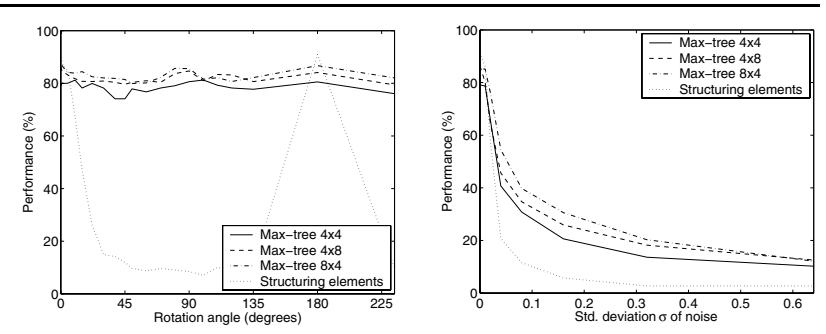

Figure 3. Performance on rotated images (left) and noisy images (right)

sured by evaluating image sets created from the original image set by adding Gaussian noise with standard deviations between $\sigma=0.01$ and 0.64 . The result of this test is shown in Fig. 3(right). On a $1 \mathrm{GHz}$ Celeron PC our methods based on the Max-tree needed 0.4 seconds to compute both pattern spectra of a typical $621 \times 501$ diatom image. Using the same image the computation time for the method using structuring elements was 1.8 seconds. Note that contrary to structuring elements methods, the computation time for our Max-tree method is independent of the number of size and shape classes.

\section{Conclusions}

Methods using pattern spectra for the computation of feature vectors are very suitable for image classification. An existing method using structuring elements was compared with a new method based on Salembier's Max-tree algorithm. For image sets without orientation or where the images were manually aligned to the have same orientation, the existing method performs better than our method. However, when rotation-invariance is desired, like in the case of images with different orientations, our Max-tree with its rotation-invariant attributes is preferable over methods based on linear structuring elements, since the latter are not invariant to rotation. Although rotation-invariance might be approximated by using linear structuring elements at many orientations, this is no option when large sets of images are used due to the sharp increase in computation time. Our method can also be used for other applications where rotation-invariance is desirable, like classification of pollen grains [8], where all grains have a more or less round shape, but whose patterns are not always symmetric. Finally, when pattern spectra with a large number of size or shape classes needs to be computed, our method is also preferable since methods based on structuring elements needs to filter an image for every class used, while our method computes a pattern spectrum in a single run. It should be noted here, that the performance of our method might be improved signifi- cantly by using other attributes. Therefore, other attributes need to be investigated.

\section{References}

[1] S. Batman and E. Dougherty. Size distributions for multivariate morphological granulometries: texture classification and statistical properties. Optical Engineering, 36(5):15181529, May 1997.

[2] E. J. Breen and R. Jones. Attribute openings, thinnings and granulometries. Computer Vision and Image Understanding, 64(3):377-389, 1996.

[3] L. Breiman. Bagging predictors. Machine Learning, 24:123140, 1996.

[4] J. M. H. Du Buf and M. M. Bayer, editors. Automatic Diatom Identification. Series in Machine Perception and Artificial Intelligence. World Scientific Publishing Co., Singapore, 2002.

[5] P. Ghosh and B. Chanda. Bi-variate pattern spectrum. In Proceedings SIBGRAPI'98, pages 476-483, Rio de Janeiro, 20-23 October 1998. IEEE Comp. Soc.

[6] P. Maragos. Pattern spectrum and multiscale shape representation. IEEE Trans. Patt. Anal. Mach. Intell., 11:701-715, 1989.

[7] R. Quinlan. C4.5: Programs for Machine Learning. Morgan Kaufmann, San Mateo, USA, 1993.

[8] O. Ronneberger, H. Burkhardt, and E. Schultz. Generalpurpose object recognition in $3 \mathrm{~d}$ volume data sets using gray-scale invariants - classification of airborne pollen-grains recorded with a confocal laser scanning microscope. In Proc. of the ICPR, Quebec, Canada, September 2002.

[9] P. Salembier, A. Oliveras, and L. Garrido. Anti-extensive connected operators for image and sequence processing. IEEE Transactions on Image Processing, 7:555-570, 1998.

[10] P. Salembier and J. Serra. Flat zones filtering, connected operators, and filters by reconstruction. IEEE Trans. Image Proc., 4:1153-1160, 1995.

[11] J. Serra. Image Analysis and Mathematical Morphology, volume 1. Academic Press, New York, 2 edition, 1982.

[12] K. Sivakumar, M. J. Patel, N. Kehtarnavaz, Y. Balgurunathan, and E. R. Dougherty. A constant time algorithm for erosions/dilations with applications to morphological texture feature computation. Real-Time Imaging, 6:223-239, 2000.

[13] E. F. Stoermer and J. P. Smol. The Diatoms: Applications for the Environmental and Earth Sciences. Cambridge University Press, 1999.

[14] E. R. Urbach and M. H. F. Wilkinson. Shape-only granulometries and grey-scale shape filters. In Proceedings of the ISMM2002, pages 305-314, 2002. 